\title{
Age and Sex Bias in Radiation Research-and How to Overcome It
}

\author{
Britta Langen \\ Department of Radiation Physics, Institute of Clinical Sciences, Sahlgrenska Cancer Center, Sahlgrenska Academy, University of \\ Gothenburg, Gothenburg, Sweden
}

B asic research is the driving force behind medical progress. As successful as this relation has been, an intrinsic dilemma persists to this day: each study design frames reality-yet the conclusions seek general validity. This dilemma crystallizes into major bias when conclusions are based on selected groups that do not represent the reality of biologic diversity. Ironically, while striving for a future of highly personalized treatments, we have overlooked the obvious features that make an individual, stratify a cohort, and influence outcome: age and sex.

A current example of this issue are molecular biomarkers that may bring the next quantum leap in clinical practice. Biomarkers such as transcripts, proteins, or metabolites can easily be sampled from blood, quantified, and used for biologic dosimetry, risk estimation for postradiation therapy diseases, or screening in radiation hazard events. Still, most studies that use novel "omics" or "nextgen" methods for screening harbor pitfalls similar to previous methodologies and neglect age and sex as important factors. This can compromise the sensitivity, specificity, and accuracy of biomarkers, leading to erroneous diagnosis and treatment planning.

Sex bias in biomedical research is not a new revelation (1). Surprisingly, it stems not only from the use of single-sex cohorts but also from omitting sex as a factor altogether. Although other fields, such as neuroscience research, have started to tackle this issue (2), it remains largely unaddressed and underrepresented in radiation biology and related medical fields. For instance, sex-specific radiation sensitivity is known in principle yet is rarely considered in study designs beyond this particular research question. The bias in our knowledge base becomes even more worrisome when considering the nonlinearity of age between humans and mice (3). Do we relate age according to sexual maturity, onset of senescence, or total life span? It is reasonable to assume that the answer is, "depending on the research question and biologic endpoint." However, this issue is usually neglected altogether and the age of the animal is chosen for purely practical reasons. Recently, research on age and sex bias has shown that radiation responses can differ largely between male and female mice, as well as between adolescent and adult specimens (4). If only one group had been used in the proteomic screening for blood-based biomarkers, the conclusions on dose-response would differ and poorly represent radiobiologic effects for other sex and

\footnotetext{
Received Feb. 11, 2019; accepted Feb. 11, 2019.

For correspondence or reprints contact: Britta Langen, Department of Radiation Physics, University of Gothenburg, Sahlgrenska University Hospital, Gula stråket 2B, SE-413 45 Gothenburg, Sweden.

E-mail: britta.langen@gu.se

Published online Mar. 8, 2018.

COPYRIGHT (c) 2019 by the Society of Nuclear Medicine and Molecular Imaging. DOI: 10.2967/jnumed.119.227470
}

age groups. Most importantly: if neglected, the bias would remain unknown and create large uncertainties that ultimately lead to avoidable risks for patients in radiotherapy and nuclear medicine.

It will be difficult to update our knowledge base to consider these basic factors systematically; in the end, a large body of evidence will still include age and sex biases. Nevertheless, the sooner we start taking action to overcome age and sex bias in our field, the less will misleading information contaminate the knowledge base. Each of us can partake in this effort according to our opportunities. For example, researchers can plan studies with male and female cohorts, principal investigators can establish such cohorts as the group standard, and manuscripts and grant applications can address these possible biases and highlight measures on how to control them. Reviewers can identify age and sex bias and consider it a methodologic limitation, and editors can establish submission forms that require disclosure of age and sex as preclinical study parameters. Lecturers can inform about these potential biases in research and raise students' awareness when working with source material. Finally, students and $\mathrm{PhD}$ candidates can take initiative and, if presented with biased data or methodologies, address age and sex as important factors.

Undoubtedly, using both male and female cohorts and different age groups in research is resource-intensive. It is paramount that funding agencies support these efforts by rewarding points for rigorous research designs that consider age and sex as essential factors. Some large international funding agencies have already started to include dedicated sections on the age and sex dimension in grant applications, but this change needs to be consistent across all funding bodies on the national and regional levels.

By committing to a higher methodologic standard, we can reduce critical bias in our field and in radiation research as a whole. Ultimately, our effort will increase the quality of diagnosis and treatment and improve the odds for therapeutic success for every patient.

\section{DISCLOSURE}

No potential conflict of interest relevant to this article was reported.

\section{REFERENCES}

1. Beery AK, Zucker I. Sex bias in neuroscience and biomedical research. Neurosci Biobehav Rev. 2011;35:565-572.

2. Will TR, Proaño SB, Thomas AM, et al. Problems and progress regarding sex bias and omission in neuroscience research. eNeuro. 2017;4(6).

3. Dutta S, Sengupta P. Men and mice: relating their ages. Life Sci. 2016;152:244-248.

4. Langen B, Spetz J, Parris TZ, et al. Age and sex bias in biomarker discovery for radiotherapy and risk estimation. In: 63rd Annual Meeting of the Radiation Research Society, Cancun, Mexico. Gothenburg, Sweden: Sahlgrenska Cancer Center; 2017. 